\title{
Logical Operator Estimation
}

Technical Report No. 134

Ulf A. Hamster

orcid: 0000-0002-0440-4868

March 11, 2021

\begin{abstract}
This report proposes an estimation method to find the unknown boolean input variable of a logical operation (AND, OR, XOR). The estimators might help to assess if the association between an input and output signal is the result of simple logical operations.
\end{abstract}

\section{Nomenclature}

\begin{tabular}{ccl}
\hline Symbol & Sets & Description \\
\hline \hline$N$ & $\mathbb{N}_{>0}$ & number of training examples \\
$k$ & $\mathbb{N}_{>0}$ & the $k$-th training example, $k=\{1,2, \ldots, N\}$ \\
$y_{k}$ & $\mathbb{B}$ & the $k$-th known boolean output signal \\
$x_{k}$ & $\mathbb{B}$ & the $k$-th known boolean input signal \\
$w$ & $\mathbb{B}$ & the unknown common boolean variable \\
$q$ & $\{a \in \mathbb{R} \mid 1 \geq a \geq 0\}$ & probability of w being true $\operatorname{Pr}(w=1)$ \\
\hline$M=2^{e}$ & $\mathbb{N}_{>0}$ & vector dimension (or embedding dimension) \\
$\mathbf{y}_{k}$ & $\mathbb{B}^{2^{e}}$ & the $k$-th known output vector of booleans \\
$\mathbf{x}_{k}$ & $\mathbb{B}^{2^{e}}$ & the $k$-th known input vector of booleans \\
$\mathbf{w}$ & $\mathbb{B}^{2^{e}}$ & the unknown common vector of booleans \\
$\mathbf{q}$ & $\{a \in \mathbb{R} \mid 1 \geq a \geq 0\}^{2^{e}}$ & vector of the probabilities $\operatorname{Pr}\left(w_{j}=1\right)$ \\
\hline
\end{tabular}

Table 1: Used symbols and notations used in this paper.

\section{Derivation of the Estimators}

\subsection{AND operator estimation}

The AND operator has four possible combinations of inputs and outputs as shown in Tab. 2. Assume the variable $\mathbf{w}$ is unknown (Tab. 3). We can certainly infer $w_{2}=1$ because $x_{2}=1 \wedge y_{2}=0$, and $w_{4}=1$ because $x_{4}=1 \wedge y_{4}=1$ but the two cases with $x_{1}=x_{3}=0$ are ambiguous because $x_{i}=0$ will always result in $y_{i}=0$ no matter the value $w_{i}$. There is a $50 \%$ chance that $w_{i}=0$ or $w_{i}=1$. Given $k=\{1,2, \ldots, N\}$ training examples with inputs $x_{k} \in \mathbb{B}$ and outputs $y_{k} \in \mathbb{B}$, the probability of $w$ being true is

$$
q=\operatorname{Pr}(w=1)=\frac{1}{N} \sum_{k=1}^{N} \overbrace{1_{x_{k}=1 \wedge y_{k}=1}^{\text {certain }}}^{\text {ambiguous }}+\overbrace{0.5_{x_{k}=0}}^{\text {and }}
$$


After estimating $q$, for a given input $x$, the output can be inferred as $\hat{y}$

$$
\hat{y}=(q>0.5) \wedge x
$$

\begin{tabular}{|cc|c|}
\hline$w$ & $x$ & $y$ \\
\hline \hline 0 & 0 & 0 \\
0 & 1 & 0 \\
1 & 0 & 0 \\
1 & 1 & 1 \\
\hline
\end{tabular}

Table 2: Truth table for $w \wedge x=y$.

\begin{tabular}{|cc|c|}
\hline$w_{i}$ & $x_{i}$ & $y_{i}$ \\
\hline \hline$w_{1}$ & 0 & 0 \\
$w_{2}$ & 1 & 0 \\
$w_{3}$ & 0 & 0 \\
$w_{4}$ & 1 & 1 \\
\hline
\end{tabular}

Table 3: Unknown $w_{i}$ for AND operations.

\subsection{OR operator estimation}

The OR operator has certain solutions for $w_{i}$ when $x_{i} \neq y_{i}$ and $x_{i}=y_{i}=0$, but is ambiguous when $x_{i}=y_{i}=1$ (Tab. 5). The estimator for $\operatorname{Pr}(w=1)$ can be formulated as follows:

$$
q=\operatorname{Pr}(w=1)=\frac{1}{N} \sum_{k=1}^{N} 1_{x_{k}=0 \wedge y_{k}=1}+0.5_{x_{k}=1}
$$

The output $\hat{y}$ can be inferred with the input signal $x$ and the estimated probability $p$

$$
\hat{y}=(q>0.5) \vee x
$$

\begin{tabular}{|cc|c|}
\hline$w$ & $x$ & $y$ \\
\hline \hline 0 & 0 & 0 \\
0 & 1 & 1 \\
1 & 0 & 1 \\
1 & 1 & 1 \\
\hline
\end{tabular}

Table 4: Truth table for $w \vee x=y$.

\begin{tabular}{|cc|c|}
\hline$w_{i}$ & $x_{i}$ & $y_{i}$ \\
\hline \hline$w_{1}$ & 0 & 0 \\
$w_{2}$ & 1 & 1 \\
$w_{3}$ & 0 & 1 \\
$w_{4}$ & 1 & 1 \\
\hline
\end{tabular}

Table 5: Unknown $w_{i}$ for OR operations.

\subsection{XOR operator estimation}

The beauty of XOR is that there is deterministic, certain solution $w=x \oplus y$. As a result the estimator is pure counting:

$$
q=\operatorname{Pr}(w=1)=\frac{1}{n} \sum_{k=1}^{n} 1_{x_{k}=0 \wedge y_{k}=1}+1_{x_{k}=1 \wedge y_{k}=0}
$$

Given the input signal $x$, and estimated probability $\operatorname{Pr}(w=1)$ of the unknown boolean $w$, the output $\hat{y}$ can be inferred as follows:

$$
\hat{y}=(q>0.5) \oplus x
$$




\begin{tabular}{|cc|c|}
\hline$w$ & $x$ & $y$ \\
\hline \hline 0 & 0 & 0 \\
0 & 1 & 1 \\
1 & 0 & 1 \\
1 & 1 & 0 \\
\hline
\end{tabular}

Table 6: Truth table for $w \oplus x=y$.

\begin{tabular}{|cc|c|}
\hline$w_{i}$ & $x_{i}$ & $y_{i}$ \\
\hline \hline$w_{1}$ & 0 & 0 \\
$w_{2}$ & 1 & 1 \\
$w_{3}$ & 0 & 1 \\
$w_{4}$ & 1 & 0 \\
\hline
\end{tabular}

Table 7: Unknown wi for XOR operations.

\section{Probability of Error}

The estimation of $q$ can be treated as two-tailed hypothesis test whether $q$ is clearly true or false.

$$
H_{0}: q=0.5 \quad H_{a}: q \neq 0.5
$$

The probability of error ( $\mathrm{p}$-value) can be computed using the binomial test or the Pearson's $\chi^{2}$ test.

\section{Serialize as Integer}

A vector of $M=2^{e}$ boolean variables $\mathbf{w}, \mathbf{x}_{\mathbf{k}}, \mathbf{y}_{\mathbf{k}} \in \mathbb{B}^{M}$ can be serialized as $e$-bit integer variable $\mathbb{N}$. The estimators are vectorizable, i.e. the probability $p_{j}=\operatorname{Pr}\left(w_{j}=1\right)$ can be estimated for each association $\left(x_{j}, y_{j}\right) \forall j=1,2, \ldots, M$ in parallel.

\section{Batch training}

Given $1>r>0$ the learning rate, $\mathbf{q}^{(l)}$ the previous probabilities, and $\mathbf{q}^{\text {new }}$ the probabilities estimated based on the $l$-th batch, the probabilities $\mathbf{q}$ are updated as follows:

$$
\mathbf{q}^{(l+1)}=(1-r) \mathbf{q}^{(l)}+r \mathbf{q}^{\text {new }}
$$

The learning rate can be set to $r=N_{B} / N$ with $N_{B}$ the batch size and $N$ the size of the training set.

\section{Conclusion and further research}

The report proposed estimators to identify patterns of logical connections between boolean inputs and outputs. The next steps are to find applications for the proposed estimators, e.g. encode batches of boolean input and output vectors into weights $q \in \mathbb{R}^{d}$ for neural networks, train masks for sparse matrices, find associations between integer data and binary data.

\section{References}

[VD09] Guido Van Rossum and Fred L. Drake. Python 3 Reference Manual. Scotts Valley, CA: CreateSpace, 2009. ISBN: 1441412697.

$[$ Har +20$]$ Charles R. Harris et al. "Array programming with NumPy". en. In: Nature 585.7825 (Sept. 2020), pp. 357-362. ISSN: 1476-4687. DOI: 10.1038/s41586020-2649-2. 
[Vir +20$] \quad$ Pauli Virtanen et al. "SciPy 1.0: fundamental algorithms for scientific computing in Python". In: Nature Methods 17.3 (Mar. 2020), pp. 261-272. ISSN: 1548-7105. DOI: $10.1038 / \mathrm{s} 41592-019-0686-2$.

\section{A Demo Implementation}

The demo code is written in Python3 [VD09] and uses the packages NumPy $\mathrm{Har}+20]$ and SciPy $\mid$ Vir $+20 \mid$. Generate toy data

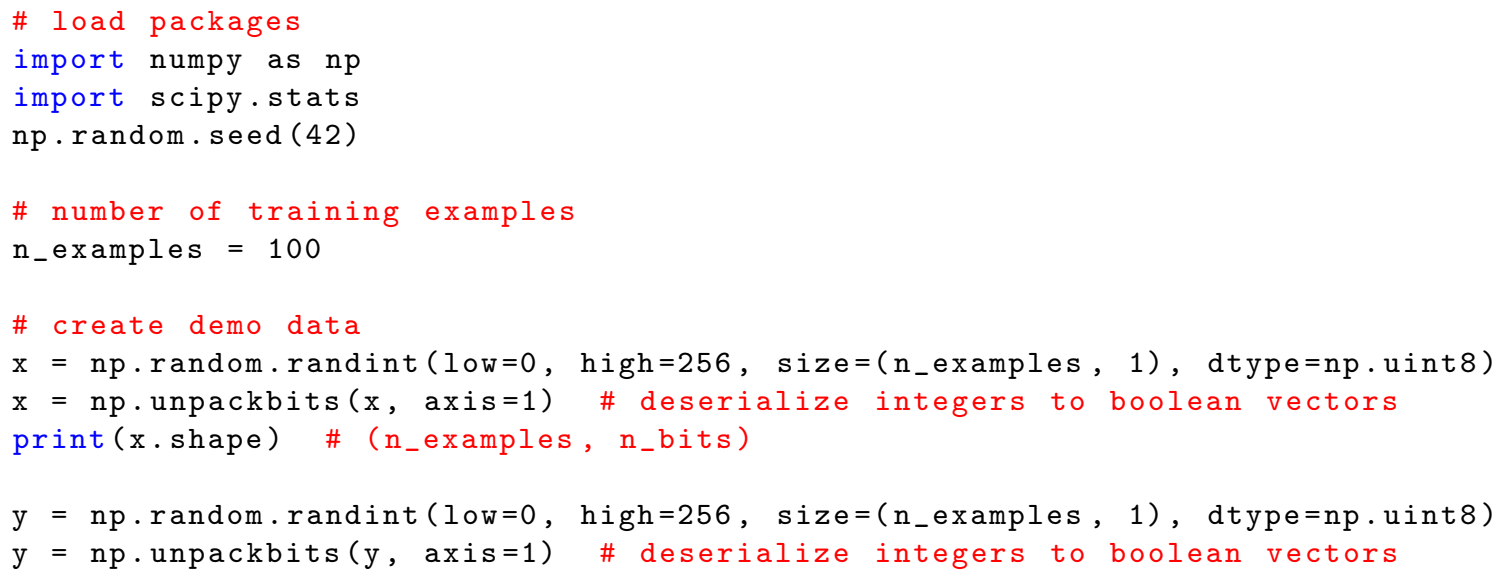

AND operator estimation

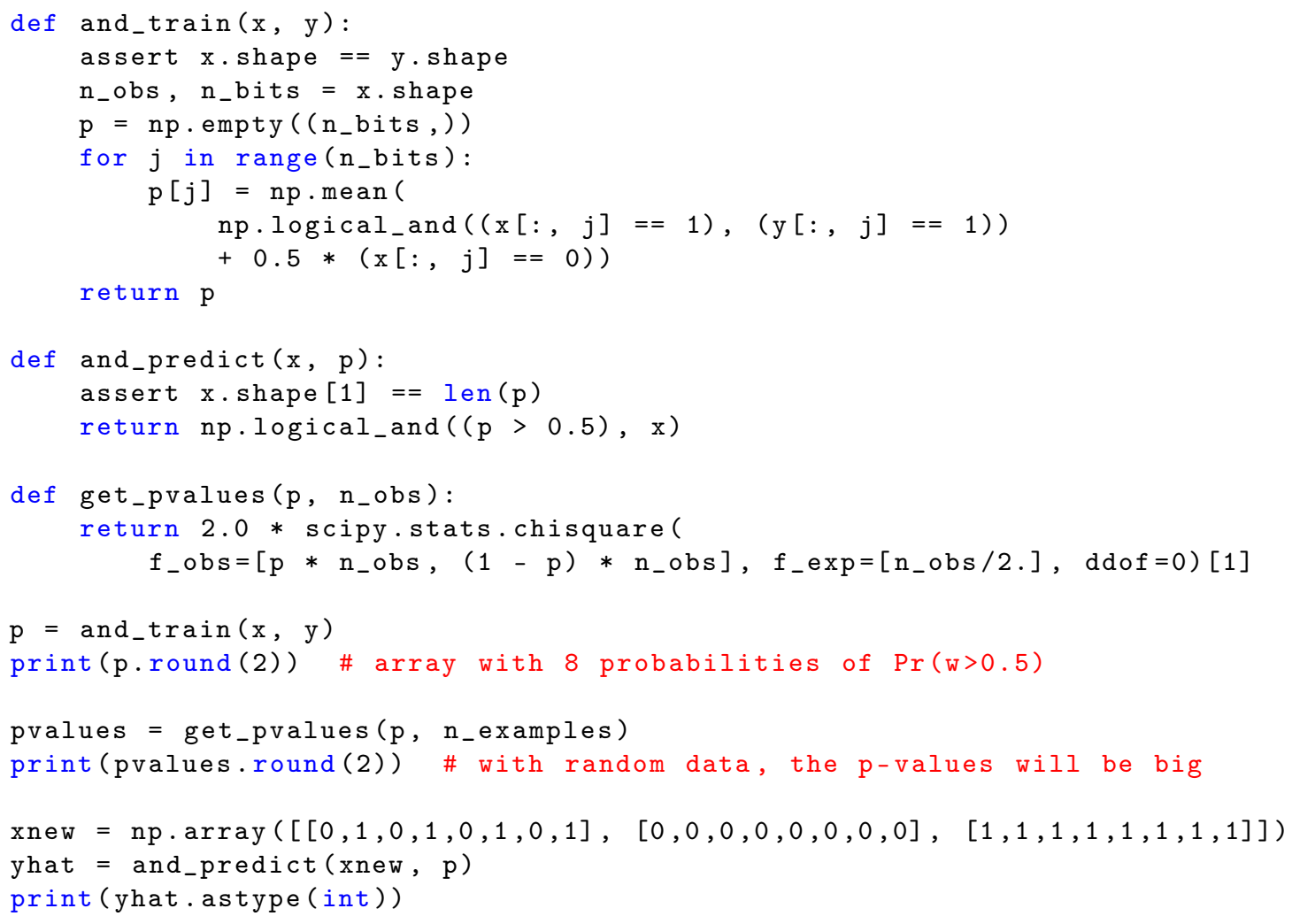

OR operator estimation

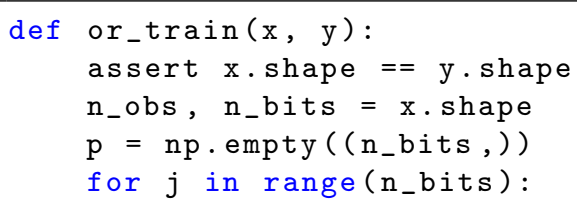




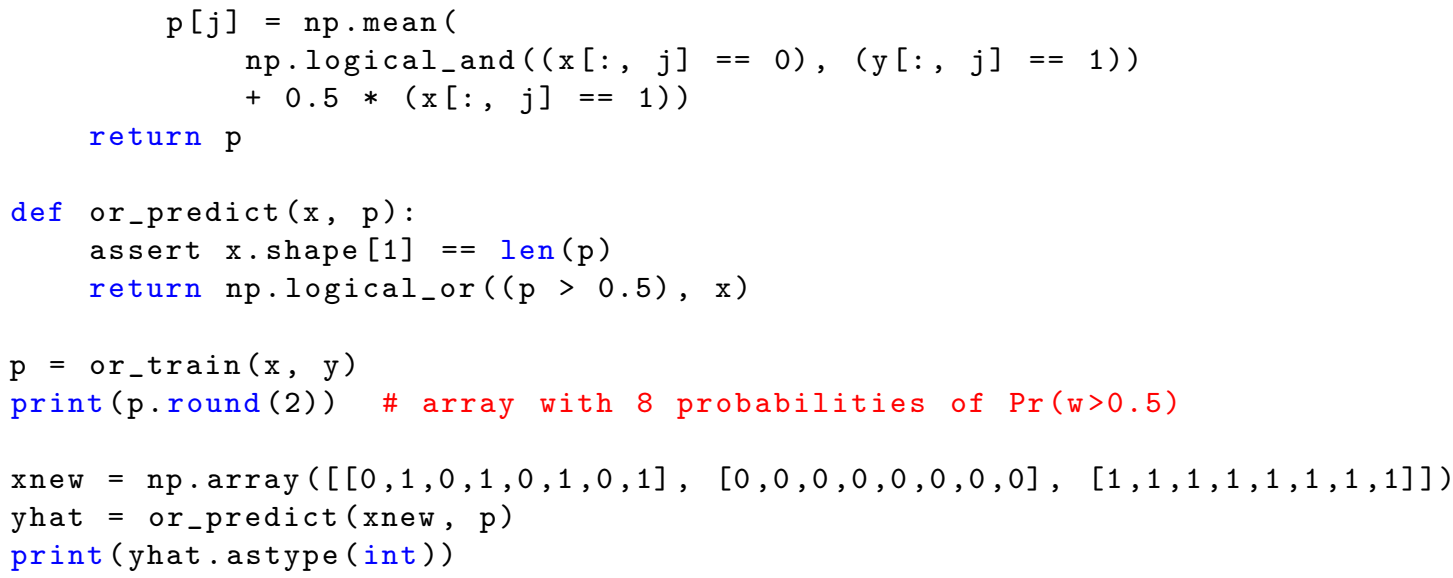

XOR operator estimation

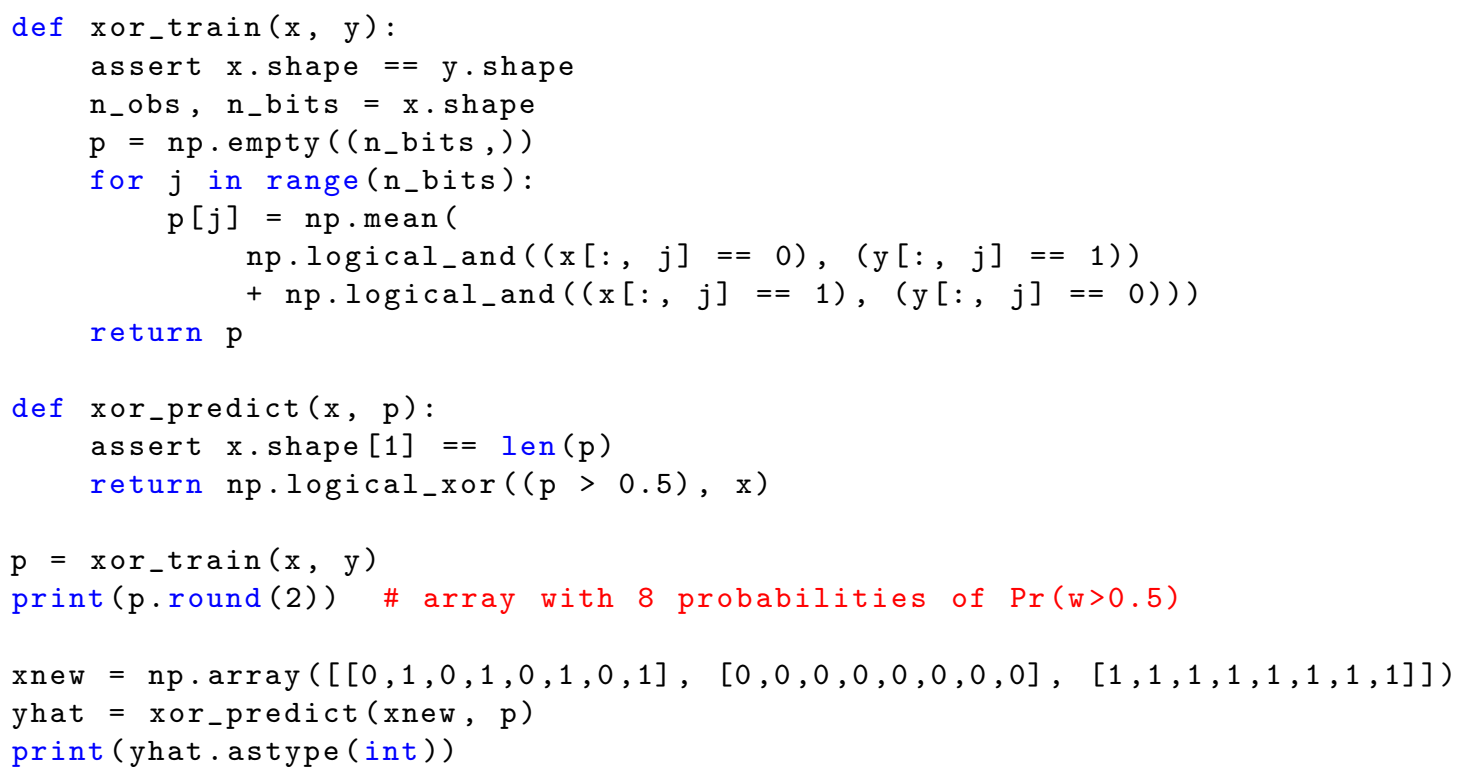

